\title{
Fault Diagnostic System for Centrifugal Milk Separator's Rotor Bearings with Vibration Isolators
}

\author{
Andrius TRUMPA, Vladas VEKTERIS, Vadim MOKŠIN, Artūras KILIKEVIČIUS
}

\begin{abstract}
The paper deals with the fault diagnostic system of centrifugal milk separator's rotor bearings with vibration dampers. The diagnostic system consists of a number of accelerometers attached to the outer ring of the upper bearing of the rotor and the separator's housing. Such a system allows measuring bearing outer ring vibrations directly and indirectly (through the housing) and at the same time it allows to evaluate the impact of bearing vibration dampers. Direct and indirect bearing vibration measurement results and the differences between them are presented in the paper as well as the relationship between the vibration acceleration and excitation force spectra.
\end{abstract}

Keywords: centrifugal milk separator; fault diagnostics; rolling element bearings; vibration damper

\section{INTRODUCTION}

Milk separation process causes vibrations that affect separator's rotor-bearing system elements, especially the rolling element bearings. One of the ways to reduce vibrations of the rotor-bearing system is to use the vibration isolators in the bearing supports. There are many technical solutions to reduce vibration transmission from one component to the other components of the machine. They are commonly referred to as various vibration dampers, for example, shock absorbers, mechanical springs, pneumatic and hydraulic devices as well as their combinations. Conventional passive vibration dampers are usually used to suppress high frequency vibrations and control the resonance, when the high level of damping is required [1-8]. They are effective only if excitation frequencies are much higher than their natural frequencies $[2,4]$. However, in practice, frequency of external excitation is varied in time, or excitation has a frequency spectrum. In these cases, the effectiveness of passive vibration dampers is reduced if the excitation frequency is close to the natural frequency.

In determining the damping parameters of the system, it is usually necessary to combine different frequency damping and generalize the effectiveness of damping system. Therefore different type vibration damping systems are used to suppress vibrations of the rotor bearings of centrifugal milk separators of different productivity. In most cases, it is visco-elastic and hysteretic damping, because active and other types of dampers are hardly applied in rotor-bearing systems of centrifugal milk separators. Such rotor-bearing systems with passive bearing vibration dampers are widely used in the dairy industry. Depending on its productivity, the separator can be equipped with single transverse vibration damping system or transverse and axial vibration damping systems [9]. The last passive systems are used in high productivity milk processing equipment (separators, purifiers and bactofuges).

Since the rotation accuracy and rotational speed are increased, vibration and noise issues related to the rotors supported by rolling bearings become extremely important. As one of the sources of vibration and noise, rolling element bearings attracted substantial attention because of their significant effects on dynamic characteristics of high- speed rotor systems, which is due to the Hertzian forces, varying compliances, internal clearances and so on [10]. This situation creates increasing interest in non-linear vibration analysis of rolling bearing-rotor systems. Rolling element bearings have very complicated dynamic behaviour due to the number of rolling elements except the fixed outer race or inner race. Not only that, but their motion behaviour is very sensitive to initial conditions also. Rotor is supported at the two ends by two ball bearings and the separator's bowl is mounted on that rotor. The bowl causes the unbalance force. Unbalance force is changed continuously with change in the speed of rotor and changes the dynamic behaviour of the whole system continuously [11]. So, it is necessary to study the effect of unbalance force on the dynamic behaviour of the rotorbearing system.

It can be stated that most of the studies were carried out with single row rolling bearings and bearing systems. However, damped double-row rolling element bearings without internal clearance used in centrifugal milk separators and other equipment require a different approach and a new type of diagnostic measurement technique with methodology for evaluation of uncertainties.

Bearings with vibration dampers are the most damageable components of centrifugal milk separators. From the fault diagnosis point of view, this rotor-bearing system is complex, because it is difficult to measure its vibrational characteristics due to the interference of vibration dampers that distort measurement results.

In order to obtain data related to the milk separator's rotor bearings condition vibration measurement sensors are used that are attached to the housing of the separator and to the rotor bearing outer ring holder [12-14]. Received input signal processing procedure has to be carefully thought out to be able to distinguish vibrations of the new bearing from those of the faulty bearing and to obtain information necessary to detect the fault. For this purpose, a methodology should be developed in order to assess the reliability of the results obtained. Diagnostics of such mechanisms is not well developed. It is therefore necessary to provide the layout of a number of sensors to separate the vibration excitation sources that act simultaneously, but are located at different points of the separator. The authors believe that it is possible to create such diagnostic system 
that would be able to distinguish the characteristics of excitation sources of the milk separator and predict the appearance of bearing faults. Such a system does not require interference in the work of the separators and can be used in their quality assessment and control during manufacturing process, in the process of fault diagnosing during the scheduled maintenance procedure and during the exploitation process.
In any case, when using fault diagnostic and monitoring system, a significant economic effect can be achieved. It was established that the mechanism operation and maintenance costs decrease by $30-40 \%$ [13]. The installation expenses of these systems are small compared with the profit - about 1:10 [14].

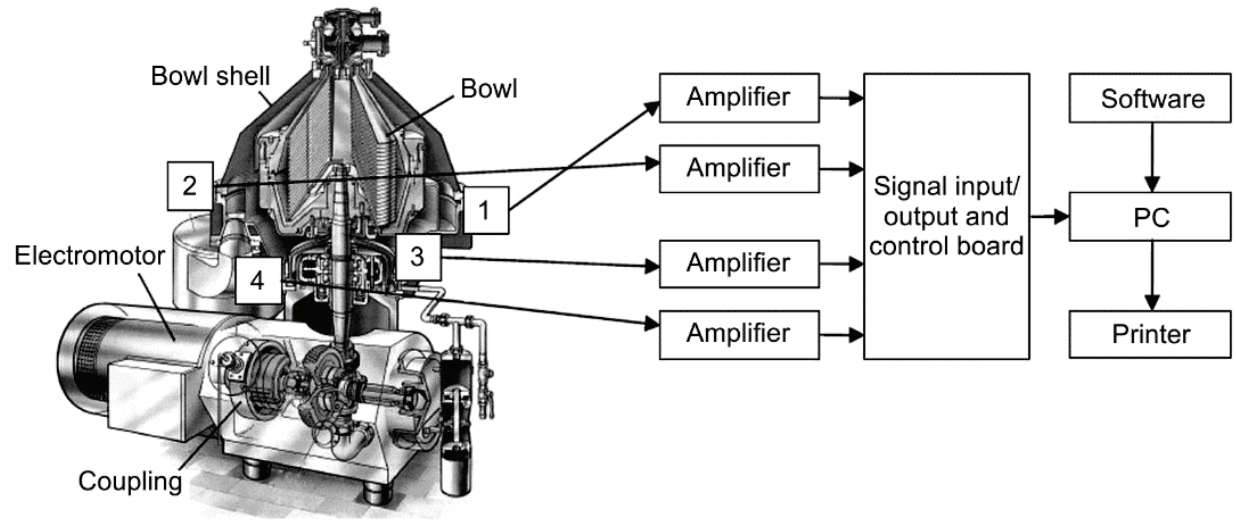

Figure 1 Diagnostic scheme: 1, 2, 3, 4 - accelerometers

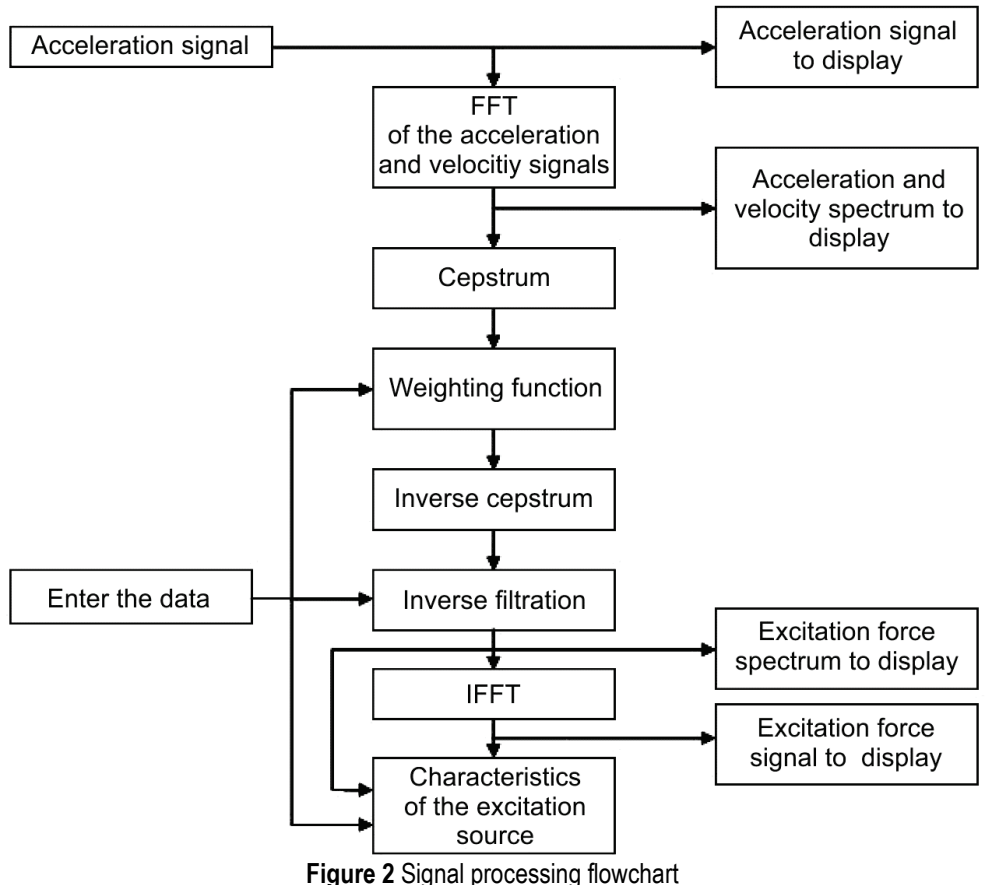

\section{DIAGNOSTIC SYSTEM AND RESULTS OF DIAGNOSTIC MEASUREMENTS}

Diagnostic scheme and arrangement of sensors is presented in Fig. 1. Four accelerometers are used to measure the vibration acceleration of the outer ring of the upper bearing and the housing of the centrifugal milk separator.

Vibration acceleration of the separator was measured using Vibration Analyser Adash 4300-VA3. The recorded data was processed and analysed with OriginLab Corporation Origin 6.1 software.

At any stage of signal processing the data can be reviewed on the PC display in order to be certain that the signals are processed correctly. The signal processing flowchart is presented in Fig. 2. The fast Fourier transformation (FFT) is applied to the vibration acceleration signal and the acceleration frequency spectrum is obtained, then the cepstrum of the signal is calculated. Cepstrum separates the "short-term" components of the signal related to the direct spreading of vibrations from the source from the "long-term" components caused by the reflection and reverberation of waves in the construction. There is a possibility to simplify the procedure of identification of the vibration source characteristics by keeping only the "short-term" components of the signal. After that, the inverse cepstrum operation is applied. An inverse filtration is used for the obtained spectrum to proceed from vibration acceleration to the excitation force. In the simplest case, this inverse 
filter has the transmission function, which is inverse to the transmission function from the excitation force at the sensor attachment point. In a general case, such inverse filter can have such a "cepstrum window" that is used for the vibration acceleration signal. In the cases when there are several excitation and measurement points, more sophisticated inverse filters can be created in order to improve the diagnosis possibilities of the system.
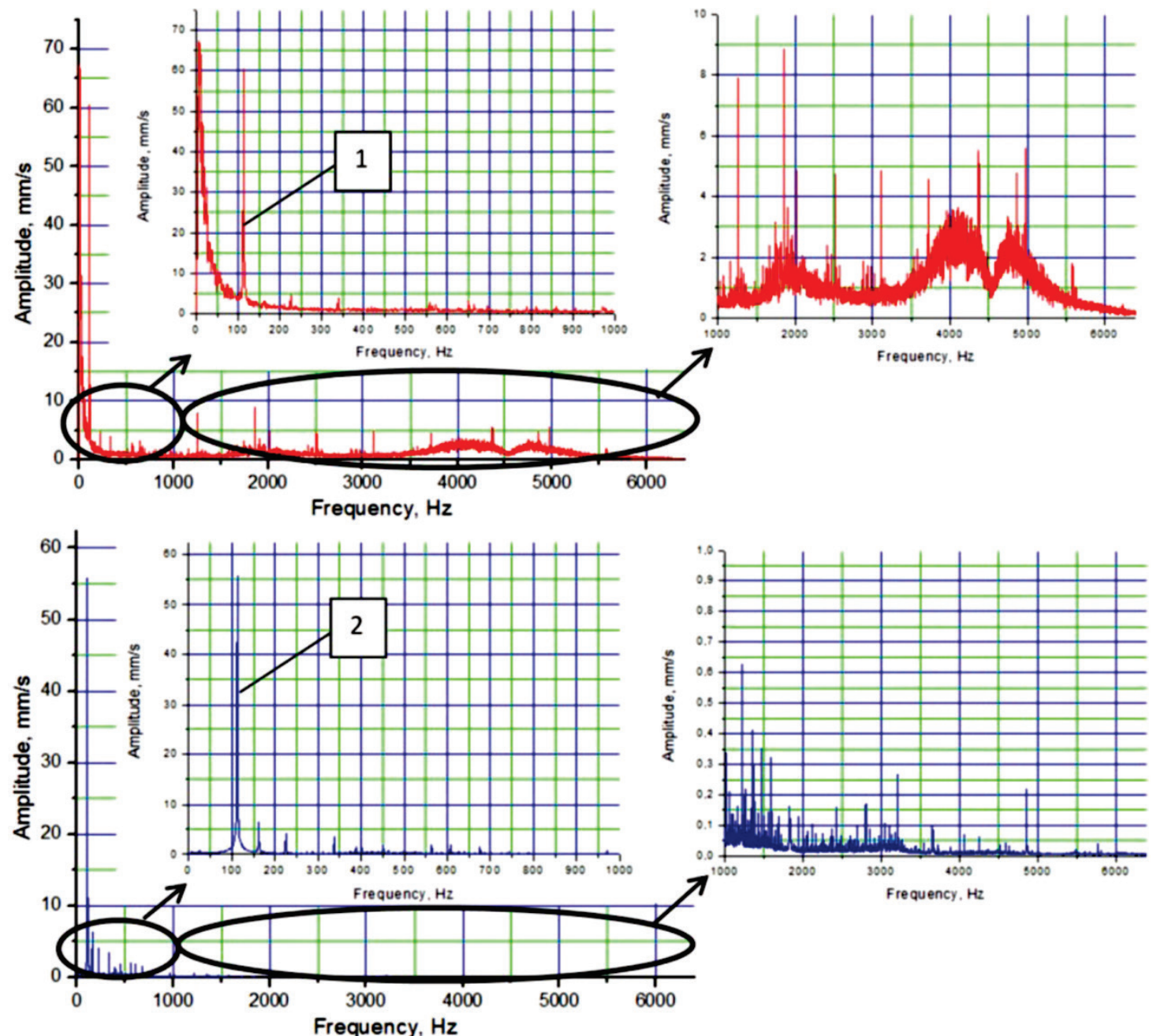

Figure 3 Vibration velocity spectra obtained from direct bearing vibration measurements: 1 - case when the faulty bearing was used in rotor-bearing system, 2 - case when the new bearing was used in rotor-bearing system

Using a suitable inverse filtering procedure one or another excitation force frequency spectrum can be obtained. This spectrum or corresponding time domain signal obtained by inverse fast Fourier transformation (IFFT) can be used for fault diagnosis, for example, making a decision about admissibility of the excitation source. These results can also be used to establish the deviation of the signal from some "reference" signal or to determine the parameters of measured signal to which permissible variation limits are assigned. Fault diagnostic system (Fig. 1, 2) was realized to detect faults of vertical rotor-bearing system equipped with vibration isolators. It is also able to establish excitation source and excitation force. Fig. 3 and 4 present the velocity signal frequency spectra obtained for the cases when the new and the faulty bearing were used to support the rotor. This data have been transformed by means of cepstral window and inverse filter determined in accordance with the transmission function results from the force to the bearing acceleration.

It can be seen from Fig. 3 that in case of direct outer ring vibration measurements the differences between velocity amplitudes of the faulty bearing and the new bearing are observed in the whole frequency range. If the vibrations of the housing were measured (Fig. 4), these amplitude differences are observed at high frequencies only, i. e. from $1000 \mathrm{~Hz}$ to $5000 \mathrm{~Hz}$. Velocity amplitudes of the faulty bearing and the new bearing obtained in direct and indirect (through housing) measurements differ about ten times. It can be stated that the vibration transfer from bearing to the housing is different for the faulty bearing and the new bearing and velocity amplitudes of the faulty bearing differ from amplitudes obtained for the new bearing at high frequencies only. Therefore, by measuring the vibrations of the separator housing decision about bearing fault can be made by high-frequency amplitudes (starting from approximately $1000 \mathrm{~Hz}$ ). Indirect diagnostic vibration measurements show significantly reduced velocity values at frequencies up to $1000 \mathrm{~Hz}$.

Fig. 5, a presents the time domain signal of accelerometer and vibration acceleration frequency spectrum obtained in the case when the rotor rotated at $6745 \mathrm{rpm}$. Fig. $5 \mathrm{~b}$ presents excitation force versus time plot and excitation force frequency spectrum obtained by diagnostic system (Fig. 2). 
It can be seen that the excitation amplitude and the excitation force calculation results have similarities with the results of faulty bearing diagnostic measurements (Fig. 5a). The milk separator excites vibrations due to the milk movement in the bowl and rotor unbalance. Therefore, direct bearing vibration measurements (of the outer ring of the bearing) reflect the condition of the bearings. To realize them design changes should be made similar to those of the experimental setup (Fig. 1) in order to carry out direct diagnostic measurements and assess the condition of the bearings.
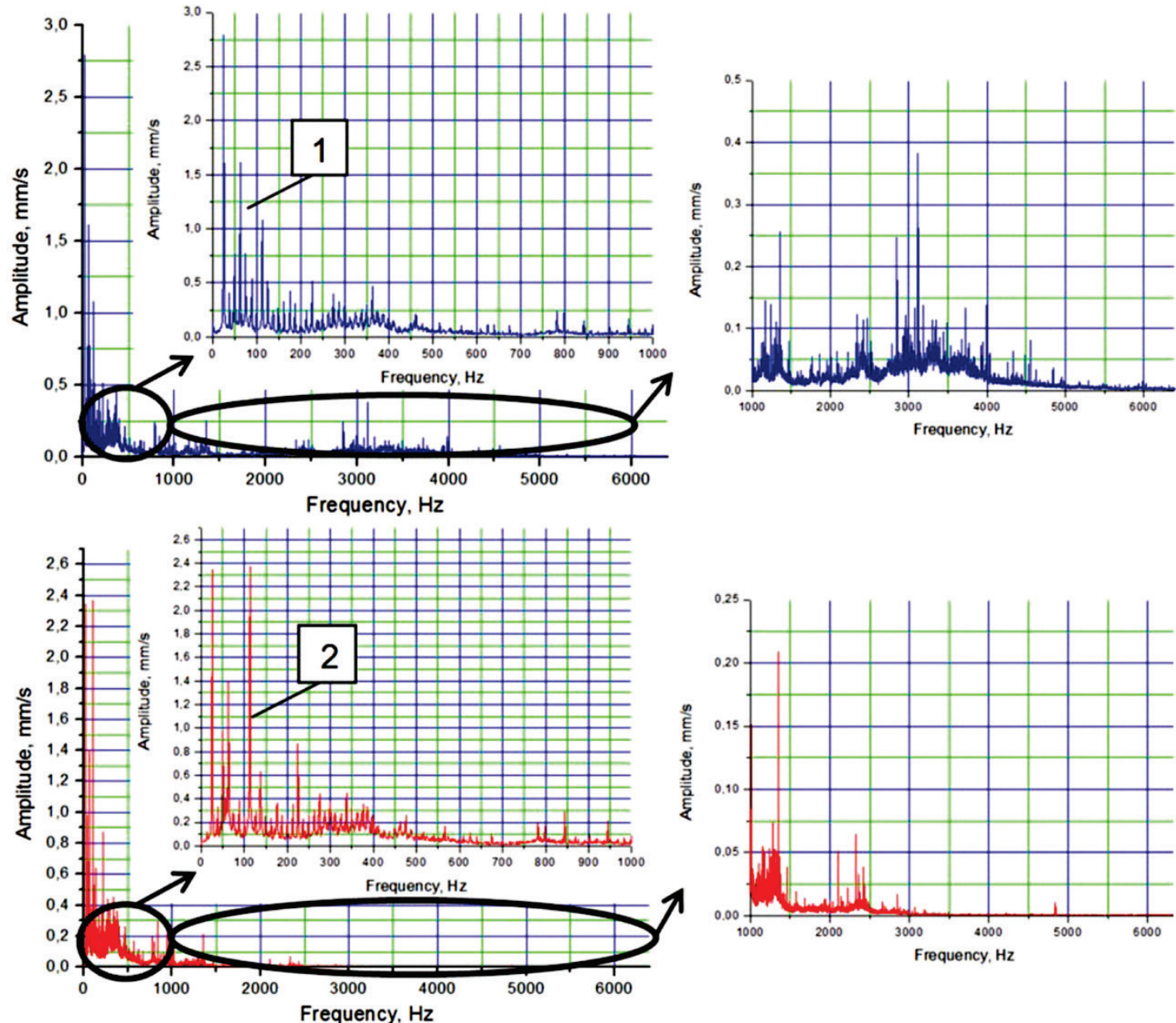

Figure 4 Vibration velocity spectra obtained from housing vibration measurements: 1 - case when the faulty bearing was used in rotor-bearing system, 2 - case when the new bearing was used in rotor-bearing system

a)

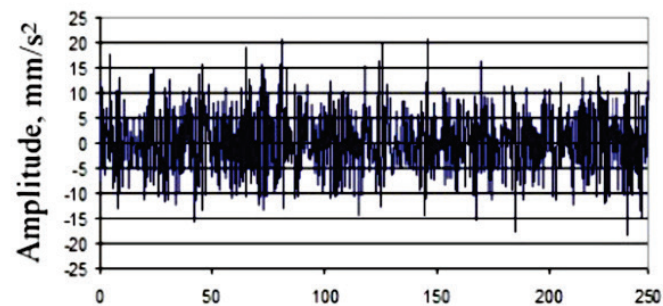

Time, ms

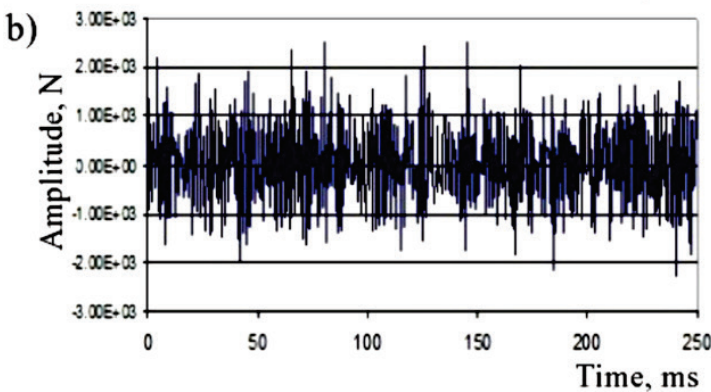

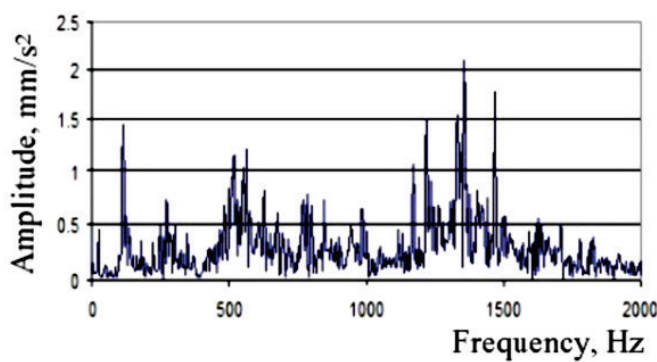

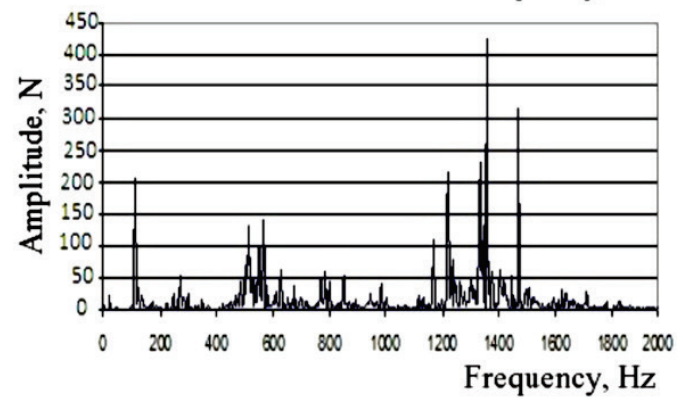

Figure 5 Vibration acceleration (a) and excitation force (b) versus time plots and their frequency spectra obtained for the case when the faulty bearing was used in rotorbearing system of the separator 


\section{CONCLUSION}

When using the fault diagnostic system, the nature of the excitation source and the excitation power can be identified and the design of the bearing unit as well as the design of the damping system can be modified.

In the purpose to increase the reliability of diagnostic measurements it is proposed to use direct rolling element bearing vibration measurement method, when the accelerometer is attached to the outer bearing ring through the connector. Direct measurement method based on the information about bearing parameters allows assessing condition of the separator's bearings in cases when the decision cannot be made based on the results of indirect vibration measurements.

\section{REFERENCES}

[1] Karnopp, D. C. (1973). Active and Passive Isolation of Random Vibration. Isolation of Mechanical Vibration Impact and Noise: A Colloquium Presented at the ASME Design Engineering Technical Conference / Cincinnati, 6486.

[2] Hansen, C. H., Snyder, S., Qiu, X., Brooks, L., \& Moreau, D. (2012). Active Control of Noise and Vibration. $2^{\text {nd }}$ ed. Boca Raton : CRC Press.

[3] Bies, D. A. \& Hansen, C. H. (2009). Engineering Noise Control: Theory and Practice. $4^{\text {th }}$ ed. Abingdon: Spon Press.

[4] Fuller, C. R., Elliott, S., \& Nelson, P. A. (1997). Active Control of Vibration. London: Academic Press. https://doi.org/10.1002/9780470172520.ch75

[5] Soong, T. T. (1990). Active Structural Control: Theory and Practice. 1990, Boston : Addison-Wesley.

[6] Rao, S. S. (1995). Mechanical Vibrations. $3^{\text {rd }}$ ed. Boston : Addison-Wesley,.

[7] White, R. G. \& Walker, J. G. (1982). Noise and Vibration. Hemel Hempstead : Ellis Horwood Ltd.

[8] Ver, I. L. \& Beranek, L. L. (2006). Noise and Vibration Control Engineering: Principles and Applications. $2^{\text {nd }}$ ed. Hoboken : John Wiley and Sons, Inc.,.

[9] Alfa Laval Self-Cleaning Hermetic Milk Separator. Lund : Alfa Laval AB, 2000.

[10] Bai, C., Zhang, H., \& Xu, Q. (2013). Subharmonic Resonance of a Symmetric Ball Bearing-Rotor System. International Journal of Non-Linear Mechanics, 50, 1-10. https://doi.org/10.1016/j.jinonlinmec.2012.11.002

[11] Yadav, H. K., Upadhyay, S. H., \& Harsha, S. P. (2013). Study of Effect of Unbalanced Forces for High Speed Rotor. Procedia Engineering, 64, 593-602. https://doi.org/10.1016/j.proeng.2013.09.134

[12] Vekteris, V., Trumpa, A., Turla, V., Sesok, N., Iljin, I., Moksin, V., Kilikevicius, A., Jakstas, A., \& Kleiza, J. (2017). An investigation into fault diagnosis in a rotor-bearing system with dampers used in centrifugal milk separators. Transactions of FAMENA, 41(2), 77-86. https://doi.org/10.21278/TOF.41207

[13] Barzdaitis, V. \& Činikas, G. (1998). Monitoring and Diagnostics of Rotor Machines. Monograph. Kaunas : Technologija, (In Lithuanian).

[14] Noremark, A. (1994). Condition Monitoring Based on Vibration Measurements. ABB STAL AB.

\section{Contact information:}

\section{dr. Andrius TRUMPA}

Vilnius Gediminas Technical University,

Department of Mechanical and Material Engineering,

J. Basanavičiaus 28, 03224 Vilnius, Lithuania

E-mail: andrius.trumpa@vgtu.It

prof. dr. habil. Vladas VEKTERIS

Vilnius Gediminas Technical University, Department of Mechanical and Material Engineering,

J. Basanavičiaus 28, 03224 Vilnius, Lithuania

E-mail: vladas.vekteris@vgtu.lt

prof. dr. Vadim MOKŠıN

Vilnius Gediminas Technical University,

Department of Mechanical and Material Engineering,

J. Basanavičiaus 28, 03224 Vilnius, Lithuania

E-mail: vadim.moksin@vgtu.lt

assoc. prof. dr. Artūras KILIKEVIČIUS

Vilnius Gediminas Technical University,

Department of Mechanical and Material Engineering,

J. Basanavičiaus 28, 03224 Vilnius, Lithuania

E-mail: arturas.kilikevicius@vgtu.lt 